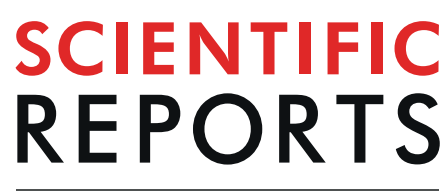

natureresearch

Received: 19 December 2018

Accepted: 25 July 2019

Published online: 06 August 2019

\section{Transcriptomic analysis of insecticide resistance in the lymphatic filariasis vector Culex quinquefasciatus}

\author{
Walter Fabricio Silva Martins ${ }^{1,2}$, Craig Stephen Wilding ${ }^{1,3}{ }^{1,}$ Alison Taylor Isaacs ${ }^{1}$, \\ Emily Joy Rippon ${ }^{1}$, Karine Megy ${ }^{4,5}{ }^{4}$ \& Martin James Donnelly ${ }^{1,6}$
}

Culex quinquefasciatus plays an important role in transmission of vector-borne diseases of public health importance, including lymphatic filariasis (LF), as well as many arboviral diseases. Currently, efforts to tackle $C$. quinquefasciatus vectored diseases are based on either mass drug administration (MDA) for LF, or insecticide-based interventions. Widespread and intensive insecticide usage has resulted in increased resistance in mosquito vectors, including $C$. quinquefasciatus. Herein, the transcriptome profile of Ugandan bendiocarb-resistant $C$. quinquefasciatus was explored to identify candidate genes associated with insecticide resistance. High levels of insecticide resistance were observed for five out of six insecticides tested, with the lowest mortality $(0.97 \%)$ reported to permethrin, while for DDT, lambdacyhalothrin, bendiocarb and deltamethrin the mortality rate ranged from 1.63-3.29\%. Resistance to bendiocarb in exposed mosquitoes was marked, with $2.04 \%$ mortality following $1 \mathrm{~h}$ exposure and $58.02 \%$ after $4 \mathrm{~h}$. Genotyping of the G119S Ace-1 target site mutation detected a highly significant association ( $p<0.0001 ; \mathrm{OR}=25$ ) between resistance and Ace1-119S. However, synergist assays using the $\mathrm{P} 450$ inhibitor PBO, or the esterase inhibitor TPP resulted in markedly increased mortality (to $\approx \mathbf{8 0 \%}$ ), suggesting a role of metabolic resistance in the resistance phenotype. Using a novel, custom $60 \mathrm{~K}$ whole-transcriptome microarray 16 genes significantly overexpressed in resistant mosquitoes were detected, with the $\mathrm{P} 450$ Cyp6z18 showing the highest differential gene expression (>8-fold increase vs unexposed controls). These results provide evidence that bendiocarb resistance in Ugandan C. quinquefasciatus is mediated by both target-site mechanisms and over-expression of detoxification enzymes.

Lymphatic filariasis (LF), is a major cause of chronic and permanent disability in tropical and subtropical regions as a result of lymphoedema, elephantiasis and hydrocele $e^{1,2}$ and is endemic in 83 countries with more than 1.2 billion people at risk of infection, especially in Southeast Asia and Africa ${ }^{3,4}$. In sub-Saharan Africa the causal agent of $\mathrm{LF}$ is the nematode Wucheraria bancrofti, which can be transmitted by both Culicine and Anopheline mosquitoes with Culex quinquefasciatus the major vector in urban settings in East Africa ${ }^{5}$.

In contrast to other vector-borne disease control programmes such as malaria and dengue that use anti-vector interventions as the major strategy, the Global Program to Eliminate LF (GPELF) is based on Mass Drug Administration (MDA) of anthelmintics to reduce W. bancrofti transmission ${ }^{6}$. Nevertheless, vector control is recommended as an intervention for LF eradication in regions where successful implementation of MDA is challenging, for instance in very remote areas, or where LF is co-endemic with loiasis which can result in adverse reactions to the drug cocktail used for $\mathrm{MDA}^{4,7}$. Modelling and field studies have shown that integration of vector

${ }^{1}$ Department of Vector Biology, Liverpool School of Tropical Medicine, Liverpool, UK. ${ }^{2}$ Laboratório de Entomologia Médica e Molecular, Departamento de Biologia, CCBS, Universidade Estadual da Paraíba, Campina Grande, Brazil. ${ }^{3}$ School of Natural Sciences and Psychology, Liverpool John Moores University, Liverpool, UK. "European Bioinformatics Institute (EMBL-EBI), Wellcome Trust Genome Campus, Hinxton, UK. ${ }^{5}$ Present address: Department of Haematology, University of Cambridge \& NHS Blood and Transplant, Cambridge, UK. ${ }^{6}$ Malaria Programme, Wellcome Trust Sanger Institute, Hinxton, UK. Walter Fabricio Silva Martins and Craig Stephen Wilding contributed equally. Correspondence and requests for materials should be addressed toW.F.S.M. (email: fabricio.martins@lstmed.ac.uk) 
control into MDA programmes can reduce the required number of chemotherapy rounds and consequently the time frame to achieve the microfilaria (MF) prevalence threshold necessary for successful interruption of LF $\operatorname{transmission}^{1,8}$.

Although vector control has successfully reduced the burden of vector-borne diseases worldwide, the recurrent and extensive application of insecticides in endemic regions has also triggered an increase in the level of insensitivity to those insecticides approved for public health ${ }^{9,10}$. In addition, the limited number of insecticides available and the occurrence of cross-resistance between different classes is especially worrying for the sustainability of vector control ${ }^{11,12}$. Consequently, identification and monitoring of resistance patterns, and understanding the underlying mechanisms is crucial for extending the lifespan of currently available insecticides, as well as for planning more effective vector control programmes.

Insensitivity to insecticides in arthropods is thought to result mainly through mutations in target-site genes and/or overproduction of detoxifying enzymes ${ }^{13,14}$. Susceptibility studies in C. quinquefasciatus from diverse geographical regions have associated two main target-site mutations to resistant phenotypes. The L1014F mutation in the voltage-gated sodium channel gene, conferring $k d r$ (knockdown resistance), has been associated with pyrethroid and DDT resistance, whilst the G119S mutation in the acetylcholinesterase (Ace-1) gene is linked to resistance to carbamates and organophosphates ${ }^{14-18}$. Metabolic resistance, which involves the over-expression, or increased catalytic capability of metabolic enzymes, is a less tractable mechanism since members of diverse gene families including carboxy/cholinesterases, glutathione S-transferases (GSTs) and cytochrome P450 monooxygenases (P450s) have previously been associated with resistance to different classes of insecticide in a range of vector species ${ }^{19-21}$. Over-expression of detoxification genes can be triggered by a range of mechanisms including gene duplication ${ }^{22}$, as observed for the resistance to organophosphates in C. quinquefasciatus mediated by esterases $^{23}$, cis-regulatory elements ${ }^{24,25}$, trans-regulatory elements, or changes in post-transcriptional repression due to differential expression of miRNAs ${ }^{26}$.

Due to the diversity of genes or gene families involved in metabolic resistance, identification of candidate genes requires an agnostic survey of the patterns of gene expression associated with resistant phenotypes. Recently, studies have applied either microarray or RNA-Seq platforms to elucidate the relationship between gene expression and insecticide resistance ${ }^{27-29}$ although to date, most of these whole-transcriptome studies in vector insects are restricted to mosquitoes of the genus Anopheles. Despite the role of Culex in transmission of several pathogens such as filarial worms and West Nile virus $(W N V)^{30}$, and reports of high levels of insecticide resistance, few studies have addressed the relative impact of metabolic resistance in C. quinquefasciatus ${ }^{31,32}$ particularly at a whole-transcriptome scale (although see ${ }^{33,34}$ ).

In addition to their role as disease vectors, C. quinquefasciatus are nuisance biters and failure to effectively control this species can lead to the perception of malaria control failure which may ultimately lead to the rejection of controls (e.g. IRS and ITNs) ${ }^{16,35}$. In this study, we report the results of C. quinquefasciatus susceptibility bioassays for six insecticides (DDT, permethrin, deltamethrin, bendiocarb, fenitrothion and lambda-cyhalothrin) from Nagongera, Tororo District, Uganda. We then report and apply a novel $8 \times 60 \mathrm{~K}$ whole-transcriptome microarray to identify candidate genes associated with bendiocarb insecticide resistance, the active ingredient used in IRS control in Uganda at the time of collection ${ }^{36}$.

\section{Materials and Methods}

Sample collection. Mosquitoes were collected in Nagongera, Tororo, Uganda $\left(0^{\circ} 47^{\prime} 48.9978^{\prime \prime}, 33^{\circ} 58^{\prime} 47.1^{\prime \prime}\right)$ between June and July 2012. Resting adult C. quinquefasciatus were collected exclusively inside houses using aspirators and transported to the insectary. From these collections, caught blood-fed females were maintained in individual Eppendorf tubes lined with moist filter paper to encourage egg laying ${ }^{37}$. From these, 64 females laid at least one egg batch. All egg batches were pooled and then floated simultaneously across five water-filled trays and emergent larvae fed on Tetramin fish food. Pupae from all trays were transferred to a single cage and adults allowed to emerge. Cages for emerged adults were changed every 3 days so that single cages contained only 3-5 day-old adults. All bioassays were performed over a single 10 day period on adults that emerged from the primary egg batches. Adult mosquitoes were fed ad libitum on 10\% glucose solution and used for insecticide susceptibility testing. Genomic DNA from each female from which egg rafts were obtained to found the colony was individually isolated using a DNeasy kit (Qiagen) then used for identification of C. quinquefasciatus using a diagnostic PCR assay ${ }^{38}$. In addition to these field-collected mosquitoes, a laboratory colony of C. quinquefasciatus from the Tropical Pesticides Research Institute (TPRI) Tanzania was used as a susceptible reference $\operatorname{strain}^{39}$ for the microarray study. The TPRI colony susceptibility to the insecticide bendiocarb (see below) was verified before the transcriptomic profiling by exposing 100 females (four replicates of $\mathrm{N}=25$ ) to bendiocarb in WHO assays (see below).

Insecticide susceptibility test. Bioassays were performed using test kits and insecticide-impregnated papers according to standard WHO methods (WHO $)^{40}$, which briefly consist of exposing mosquitoes to insecticide impregnated papers fitted inside plastic tubes $(125 \mathrm{~mm} \times 44 \mathrm{~mm})$ for 1 hour. Mosquitoes' susceptibility is assessed by the mortality rate after a 24 hour recovery period. For each insecticide tested, mosquitoes were assayed in four replicates, performed on consecutive days, using 25 non-blood fed females (3-5 day-old) emerged from the F1 generation. Mosquito rearing and bioassays were conducted under insectary conditions: $27^{\circ} \mathrm{C}+2{ }^{\circ} \mathrm{C}, 75 \% \pm 10 \%$ relative humidity and $12 \mathrm{~h}: 12 \mathrm{~h}$ light:dark cycles. Tests were performed with papers impregnated with diagnostic concentrations of six insecticides: DDT (4\%), permethrin $(0.75 \%)$, fenitrothion (1\%), lambda-cyhalothrin $(0.05 \%)$, bendiocarb $(0.1 \%)$ and deltamethrin $(0.05 \%)$. Mosquitoes were exposed for $1 \mathrm{~h}$, with the exception of bendiocarb and deltamethrin where, following the results of initial $1 \mathrm{~h}$ exposures, additional four-hour exposures were used to increase the discrimination between resistant and unexposed sympatric mosquitoes. Control assays were performed with 25 mosquitoes exposed to non-insecticide treated papers. 
(a)

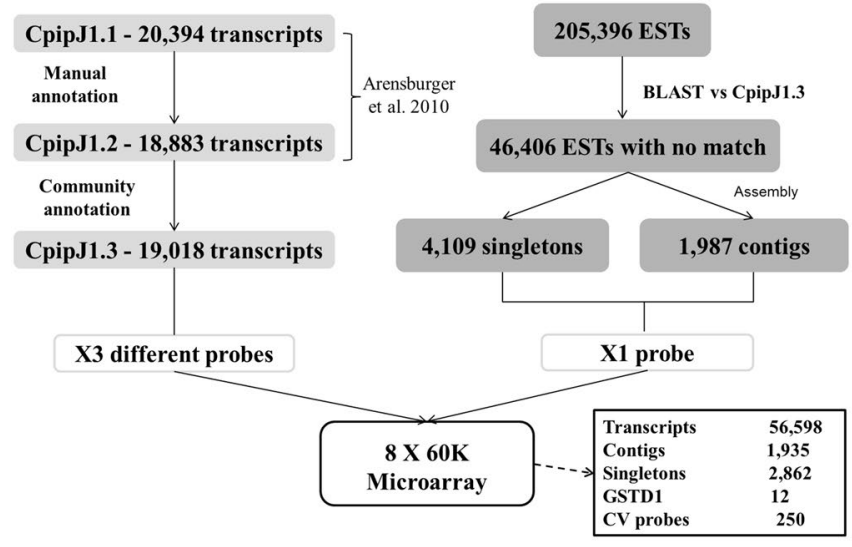

(b)

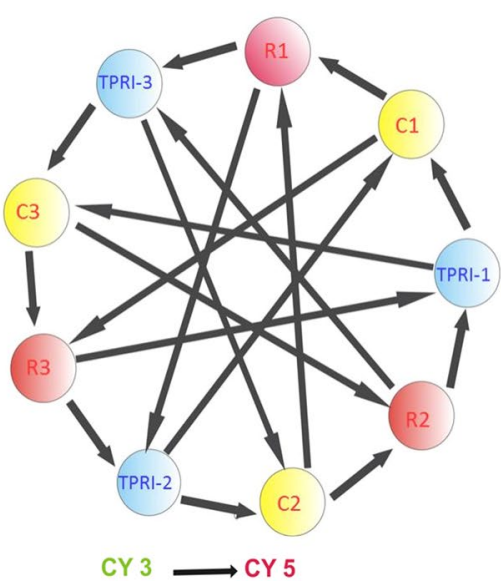

Figure 1. Overview of Culex quinquefasciatus whole-transcriptome analysis. (a) Design of the $8 \times 60 \mathrm{~K}$ Agilent microarray. CpipJ1: consensus gene set of the automated gene prediction from the C. quinquefasciatus Johannesburg strain genome sequence. EST: expressed sequence tags. GSTD1: Glutathione S transferase D1. CV probe: coefficient of variation. (b) Interwoven hybridization loop design for comparison between bendiocarb exposed and non-exposed Ugandan field-collected mosquitoes and the TPRI susceptible strain. Circles represent pools of 10 females. C: Uganda non-exposed mosquitoes (sympatric control), R: Uganda Resistant mosquitoes, TPRI (Tropical Pesticides Research Institute): C. quinquefasciatus susceptible strain from Tanzania.

Insecticide exposed mosquitoes were then transferred to clean holding tubes and provided with $10 \%$ glucose for a 24-hour period after which mortality was recorded, with dead (susceptible) mosquitoes collected and individually stored on silica gel (for downstream DNA analyses) whilst alive (resistant) mosquitoes had a hind leg removed (stored on silica) and the whole body stored in RNAlater (Sigma Aldrich). Specimens preserved on silica were used for DNA genotyping for the G119S mutation in Ace-1 while RNAlater was used to preserve RNA integrity before total RNA extraction, as described below. RNAlater stored mosquitoes were initially held overnight at $4^{\circ} \mathrm{C}$ to allow the solution to penetrate the carcass before storage at $-20^{\circ} \mathrm{C}$ until.

Synergist assays. Synergist tests were carried out using mosquitoes from the same generation utilised in the standard insecticide resistance assays following the procedure described above with an additional pre-exposure to three synergist compounds in order to investigate the potential mechanisms of metabolic resistance for bendiocarb and deltamethrin insecticides. For each synergist assay, four batches of 20-25 mosquitoes were either pre-exposed to impregnated papers $(12 \mathrm{~cm} \times 15 \mathrm{~cm}$, Whatman grade no. filter paper) with $4 \%$ PBO (piperonyl butoxide - CYP450 synergist), 10\% TPP (triphenyl phosphate - esterase synergist) or 8\% DEM (diethyl maleate GST synergist) for one hour, then exposed to bendiocarb $(0.1 \%)$ or deltamethrin $(0.05 \%)$ for four hours, followed by a $24 \mathrm{~h}$ recovery period. Synergist only controls were run simultaneously.

Ace-1 genotyping of bendiocarb phenotyped mosquitoes. Prior to microarray analysis all mosquitoes were genotyped for the G119S mutation in Ace-1. DNA was isolated from the amputated hind leg of resistant mosquitoes with $50 \mu \mathrm{l}$ of $10 \%$ Chelex 100 and $2 \mu \mathrm{l}$ of proteinase $\mathrm{K}(10 \mathrm{mg} / \mathrm{mL})$. The homogenate was incubated at $94^{\circ} \mathrm{C}$ for $30 \mathrm{~min}$ followed by centrifugation at $6,000 \mathrm{rpm}$ for $10 \mathrm{~min}$ to collect the supernatant.

A TaqMan assay, designed to detect the G119S mutation in the acetylcholinesterase gene ${ }^{41}$, was utilised for genotyping with reaction mixtures composed of $1 \mu \mathrm{l}$ of genomic DNA, 1x SensiMix II probe, $400 \mathrm{nM}$ of each primer and $100 \mathrm{nM}$ of each probe in a final volume of $50 \mu \mathrm{l}$. Thermocycling was performed on the Stratagene MX3005P and consisted of $95^{\circ} \mathrm{C}$ for $10 \mathrm{~min}$ and 40 cycles of $92^{\circ} \mathrm{C}$ for $15 \mathrm{sec}$ and $60^{\circ} \mathrm{C}$ for $1 \mathrm{~min}$ with endpoint discrimination.

Microarray. $8 \times 60 \mathrm{~K}$ microarray construction and study design. The pattern of gene expression of bendiocarb resistant mosquitoes was investigated using a custom designed C. quinquefasciatus whole genome oligonucleotide microarray (Agilent ID 039759). An $8 \times 60 \mathrm{~K}$ microarray format with 60 -mer oligonucleotide probes was designed to cover a variety of targets using eArray (http://earray.chem.agilent.com/earray/). The majority of this array encompassed three probe replicates (56,598 probes) for each of the 19,018 transcripts in the CpipJ1.3 gene build $^{42}$. Additionally, we downloaded 205,396 ESTs from VectorBase. From these, we identified 1,987 contigs and 4,109 singleton sequences and designed a single probe for each (from these 1,987 contigs, only 1,935 had probes successfully designed and from 4,109 singletons 2,862 had successful probes designed). Additionally, three probe replicates were designed to each of four alternative GST transcripts not annotated in VectorBase ${ }^{43}$, and 25 additional replicated probe groups (10 replicates) to allow estimation of reproducibility (CV (coefficient of variation probes) (Fig. 1a). Nevertheless, it is important to bear in mind that the current array version encompassed genomic information exclusively from the CpipJ1.3 annotation and may therefore lack the resolution to pinpoint 


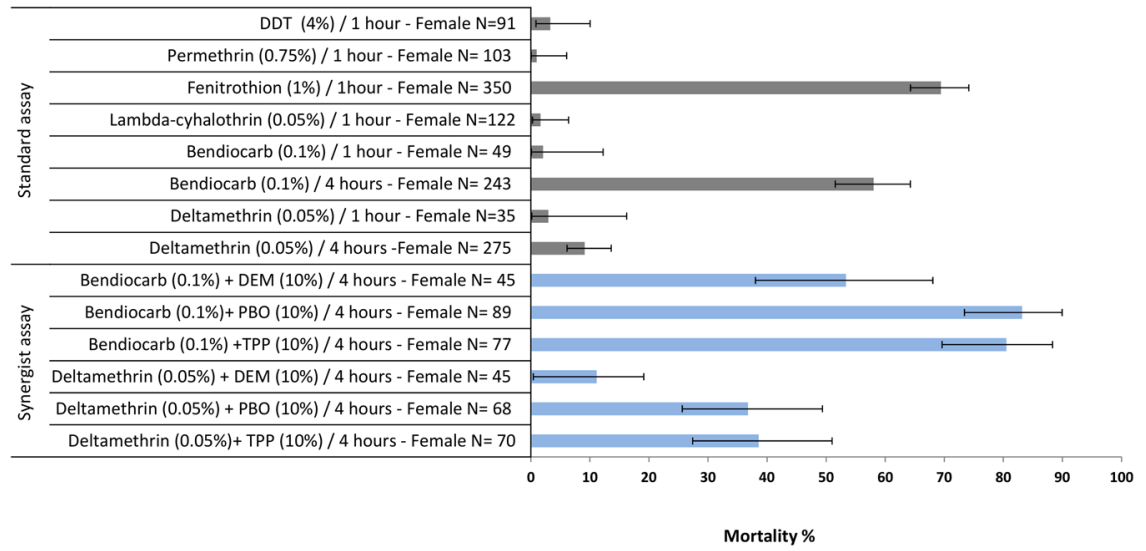

Figure 2. Insecticide susceptibility status of C. quinquefasciatus from Tororo (Uganda). Bioassay results following exposure to WHO insecticide treated papers at standard conditions and effect of insecticide synergists on the susceptibility status. Grey and blue bars represent WHO standard and synergists bioassay, respectively. Error bars represent 95\% CI. PBO: piperonyl butoxide, DEM: diethyl maleate, TPP: triphenyl phosphate.

specific gene sets linked to geographic background. Full details of the array design are given in ArrayExpress (http://www.ebi.ac.uk/arrayexpress/) with the accession number A-MTAB-649.

From the six insecticide susceptibility test groups, we chose bendiocarb selected mosquitoes for the microarray analysis since we observed only moderate mortality 58.02\% (95\% CI-confidence interval 51.53-64.25\%) for this insecticide following $4 \mathrm{~h}$ exposure (Fig. 2) thereby allowing clear discrimination between resistant and unexposed sympatric mosquitoes and we also detected an increase in mortality in the presence of two synergists (See Fig. 2; TPP and PBO), indicating the likely involvement of metabolic resistance. Three experimental conditions were employed: Tororo_Resistant (following $4 \mathrm{~h}$ bendiocarb exposure), Tororo_Control (4h exposure to control papers) and TPRI_Control (4h exposure to control papers). All mosquitoes were wildtype 119G homozygotes for Ace-1. Comparison between the bendiocarb selected samples and controls (Tororo_Control and TPRI_Control) was performed on three RNA pools per group using an interwoven loop design (Fig. 1b) as described by Vinciotti et al. ${ }^{44}$.

RNA extraction, labelling and hybridization. Total RNA was isolated from three pools of 10 female mosquitoes for each group using the RNAqueous-4PCR kit (Ambion) according to the manufacturer's instructions. Total RNA quantity was assessed using a Nanodrop spectrophotometer and RNA quality assessed on an Agilent Bioanalyzer. Each pool of RNA was individually labelled with Cyanine-3 and Cyanine-5 (Cy3 and Cy5) using the Low input Quick Amp Labelling Kit (Agilent Technologies) followed by purification through Qiagen RNeasy Columns (Qiagen) with quality and quantity checked using a Nanodrop and Bioanalyzer, respectively.

Before hybridization, $300 \mathrm{ng}$ of Cy3 and Cy5 labeled cRNA was fragmented using the gene expression hybridization kit (Agilent) in a total volume of $25 \mu$ including $5 \mu$ l of $10 \mathrm{x}$ blocking agent and $1 \mu \mathrm{l}$ of $25 \mathrm{x}$ fragmentation buffer. The fragmentation reaction was incubated at $60^{\circ} \mathrm{C}$ for $30 \mathrm{~min}$, then chilled on ice for $2 \mathrm{~min}$ before addition of $25 \mu \mathrm{l}$ of 2 x GE hybridization buffer Hi-RPM. Each array was hybridized using $45 \mu \mathrm{l}$ of the fragmentation solution for 17 hours at $65^{\circ} \mathrm{C}$ and $10 \mathrm{rpm}$. After hybridization, arrays were washed with wash buffers 1 and 2 for $1 \mathrm{~min}$ each, followed by acetonitrile for $10 \mathrm{sec}$ and finally fixation solution for $30 \mathrm{sec}$. Arrays were scanned using the microarray scanner system (Agilent Technologies) and feature extraction performed using Feature Extraction software (Agilent Technologies) according to the manufacturer's recommendations. All arrays passed the Agilent quality control with QC score $\geq 10$.

Data analysis. All microarray data analysis was performed in R version R.3.0.1 $1^{45}$. Array normalization was carried out using the Limma 3.2.3 package ${ }^{46}$ and data analysis performed using MAANOVA software version $3.0^{47}$ to detect overall differential levels of gene expression across the three treatment groups. The top overexpressed genes were selected after an ANOVA F-test based upon a false discovery rate (FDR) decision criteria of $\log _{10}$ $(Q$ value $)>2.5$. Within this subset of significantly differentially expressed probes, those that were significantly overexpressed in the Bendiocarb-exposed mosquitoes were pinpointed by comparing expression pattern in all pair-wise comparisons (see Table 1).

Additionally, the pattern of differential transcript level was also characterized through pair-wise comparisons between Ugandan resistant, sympatric control and the TPRI strain using a Student's t-test with genes considered differentially overexpressed where $P<0.05$.

Functional characterization of differently expressed transcripts detected from the ANOVA and pair-wise comparison were then submitted to a Gene Ontology (GO) analysis to classify probes on their GO categories (cellular components, biological process and molecular functions). For this, a sub-set of overexpressed probes were selected based on the threshold of $\log _{10}$ (Q value) $>2$ of the ANOVA analysis to capture a broader selection of highly overexpressed probes. This was then submitted to VectorBase for functional annotation (GO term identification) using the Biomart tool. The REVIGO web server ${ }^{48}$ was employed to summarize and visualize the distinct GO terms identified. Relatedness among GO terms was assessed using the uniqueness method, followed 


\begin{tabular}{|c|c|c|c|c|}
\hline \multirow[b]{2}{*}{ Gene } & \multirow[b]{2}{*}{ Transcript } & \multirow[b]{2}{*}{ GO } & \multicolumn{2}{|c|}{ log Fold-Change (-log Q value) } \\
\hline & & & $\begin{array}{l}\text { Ugandan Bendiocarb } \\
\text { vs TPRI }\end{array}$ & $\begin{array}{l}\text { Ugandan Sympatric } \\
\text { vs TPRI }\end{array}$ \\
\hline CPIJ020018-RA & cytochrome P450 6Z18 & monooxygenase activity & $3.13(2.80)$ & $2.59(2.80)$ \\
\hline CPIJ005900-RA & cytochrome P450 6N23 & monooxygenase activity & $2.13(2.80)$ & $1.87(2.80)$ \\
\hline CPIJ003654-RA & electron transfer & electron carrier activity & $2.36(2.58)$ & $2.23(2.58)$ \\
\hline CPIJ010823-RA & serine protease 27 precursor & serine-type endopeptidase activity & $4.41(2.53)$ & $3.86(2.44)$ \\
\hline CPIJ013083-RA & brain chitinase and chia & $\begin{array}{l}\text { hydrolase activity, hydrolyzing } \\
\text { O-glycosyl compounds }\end{array}$ & $1.31(2.44)$ & $0.93(2.38)$ \\
\hline CPIJ016928-RA & 15.4 kda salivary peptide & No information in VectorBase & $2.99(2.44)$ & $2.73(2.42)$ \\
\hline CPIJ004984-RA & serine proteases $1 / 2$ precursor & serine-type endopeptidase activity & $4.26(2.44)$ & $3.60(2.40)$ \\
\hline CPIJ011746-RA & R2D2 & double-stranded RNA binding & $1.89(2.41)$ & $1.72(2.40)$ \\
\hline CPIJ004019-RA & saccharopine dehydrogenase & oxidoreductase activity & $1.47(2.44)$ & $1.05(2.30)$ \\
\hline CPIJ004019-RA & $\begin{array}{l}\text { saccharopine dehydrogenase } \\
\text { domain }\end{array}$ & oxidoreductase activity & $1.24(2.40)$ & $1.11(2.40)$ \\
\hline CPIJ002104-RA & $\begin{array}{l}\text { plasma alpha-L-fucosidase } \\
\text { precursor }\end{array}$ & catalytic activity & $1.32(2.44)$ & $0.90(2.26)$ \\
\hline CPIJ015009-RA & histone $\mathrm{H} 3.3$ type 2 & DNA binding & $1.32(2.40)$ & $1.13(2.38)$ \\
\hline CPIJ000182-RA & $\mathrm{N}$-acetylneuraminate lyase & catalytic activity & $2.44(2.42)$ & $1.76(2.26)$ \\
\hline CPIJ011590-RA & $\begin{array}{l}\text { conserved hypothetical } \\
\text { protein }\end{array}$ & No information in VectorBase & $0.87(2.37)$ & $0.81(2.38)$ \\
\hline CPIJ019290-RA & DNA ligase 4 & DNA binding & $1.22(2.34)$ & $1.20(2.35)$ \\
\hline CPIJ016792-RA & hypothetical protein & No information in VectorBase & $3.39(2.38)$ & $2.94(2.29)$ \\
\hline
\end{tabular}

Table 1. Top differentially expressed genes from microarray analysis comparing Uganda resistant and sympatric controls compared to TPRI susceptible strain.

by clustering of GO terms with closer semantic similarity. Significantly enriched GO terms were calculated in David $^{49,50}$.

RT-qPCR validation. Reverse-transcription quantification PCR (RT-qPCR) using cDNA synthetized from the same pools of total RNA applied for microarray hybridization were used to confirm the expression profile of three out of 16 top candidate gene identified by the microarray (CPIJ020018 [=Cyp6z18], Cyp6n23, R2D2 Table 1) using two housekeeping genes - $40 S$ ribosomal protein $S 3$ and $\beta$-tubulin - as endogenous controls. Specific primers to amplify PCR fragments with size ranging from 107 to $176 \mathrm{bp}$ (see Supplementary Table S1) were designed using primer3 software ${ }^{51}$; however, only for four genes (Cyp6z18, R2D2, 40S ribosomal protein S3 and $\beta$-tubulin) was it possible to design primers spanning exon junctions (primers for Cyp6n23 were exonic only). Specificity of primer sets was verified by identification of a single symmetrical amplicon peak following melting curve analysis. Additionally, PCR efficiency was verified using a 10-fold serial dilution of standard cDNA with only primer sets with an efficiency ranging from 90 to $110 \%$ taken forward for RT-qPCR reactions.

Two technical replicates of all RT-qPCR reactions were carried out for each gene in a total volume of $20 \mu \mathrm{l}$ including $1 \mu \mathrm{l}$ of cDNA (1:4 stock diluted), $10 \mu \mathrm{l}$ of Brilliant II SYBR ${ }^{\circledR}$ master mix (Agilent Technologies) and 100 $\mathrm{nm}$ of each forward and reverse primer. Amplification was conducted under standard qPCR reaction conditions on the Mx3500P qPCR system (Agilent Technologies). Gene expression quantification of the three selected genes was assessed according to the $\Delta \Delta^{\mathrm{Ct}} \operatorname{method}^{52}$.

Manual annotation of the CPIJ020018 gene region. During qPCR primer design for the top candidate gene CPIJ020018 (annotated as Cyp6z16 in VectorBase CpipJ1.3 assembly \#1, annotation 1.3), we concluded that the available, automated gene annotation of this particular gene was unreliable. The genomic sequence of this region in VectorBase includes a region of about 810 bp in supercontig 3.2948 with no nucleotide sequence information, which spanned the automated annotation of Cyp6z16. We suspected additional coding sequence to lie within this region. To confirm this, we designed primers to span the complete region and amplified a $4.7 \mathrm{~kb}$ region covering the full length of the candidate gene genomic sequence.

PCR reactions to amplify the Cyp6z16 genomic region were conducted in a final volume of $20 \mu \mathrm{l}$ including $40 \mathrm{ng}$ of genomic DNA, $1 \mathrm{x}$ Phusion HF buffer, $200 \mu \mathrm{M}$ each dNTP, $0.5 \mu \mathrm{M}$ each primer Cx_6Z16F and Cx_6Z16R (see Supplementary Table S2) and $0.02 \mathrm{U} / \mu$ l Phusion DNA polymerase. Reaction conditions were $98^{\circ} \mathrm{C}$ for $30 \mathrm{sec}, 30 \mathrm{cycles}$ of $98^{\circ} \mathrm{C}$ for $10 \mathrm{sec}, 62^{\circ} \mathrm{C}$ for $30 \mathrm{sec}$ and $72^{\circ} \mathrm{C}$ for $3 \mathrm{~min}$, with a final extension of $72^{\circ} \mathrm{C}$ for $5 \mathrm{~min}$. PCR products were purified using the GeneJET PCR purification kit (Thermo Scientific) then cloned into pJET1.2 PCR vector (Thermo Scientific). Finally, the full length of cloned PCR product after plasmid purification using the GeneJET Plasmid Miniprep Kit (Thermo Scientific) was sent to Source BioScience, UK for Sanger sequencing using nine internal primers (see Supplementary Table S2). Sequence traces were analyzed using CodonCode Aligner version 4.2.2. Following removal of vector sequences a single contig was built from overlapping sequences. This contig sequence was then used for gene structure prediction and transcript annotation using the Augustus web interface ${ }^{53}$. 
(a)

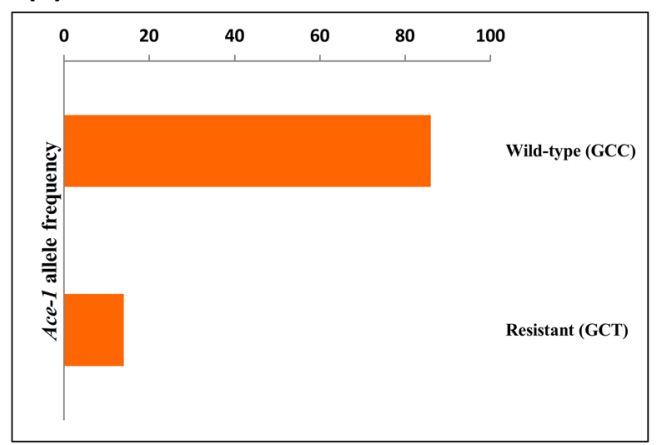

(b)

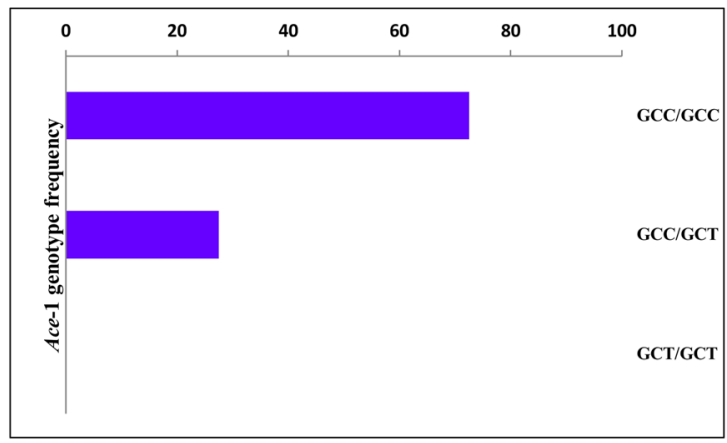

(c)

\begin{tabular}{llllllll}
\hline & \multicolumn{9}{c}{ Genotype } & & & \\
\cline { 2 - 7 } & GG & GS & SS & $\boldsymbol{P}$ & OR & LCl & UCl \\
\hline Alive & 46 & 34 & 0 & 0.001 & 25 & 3.37 & 186 \\
Dead & 46 & 1 & 0 & & & & \\
\hline
\end{tabular}

Figure 3. Ace1-119S allele and bendiocarb association test in C. quinquefasciatus. (a) Ace-1 allelic (b) genotypic frequencies (c) association of the Ace-1 genotype and bendiocarb (0.1\%)/4 hours resistant phenotype.

Transgenic expression in Drosophila flies. The full-length sequence of Cyp6z18 was codon-optimised by Genscript (Piscataway, NJ, USA) for Drosophila and synthesised with EcoRI-XbaI sites then cloned into the pUAST.attB vector (provided by Dr J Bischof, University of Zurich). Transformation was performed using the PhiC31 system with plasmids injected into the germline of $D$. melanogaster with a chromosome 2 attP landing site (y w M(eGFP, vas-int, dmRFP)ZH-2A; P\{CaryP $\}$ attP40) by the University of Cambridge Fly Facility. A single transgenic line was generated and balanced. To induce expression of Cyp6z18, flies were crossed to the Act5C-GAL4 strain (y1 w*; P (Act5C-GAL4-w) E1/CyO,1;2) (Bloomington Stock Center, IN, USA). For each treatment, Cyp6z18 transgenic or untransformed controls, three crosses of 12-15 females to 6-7 Act5C-GAL4 males were performed. Shortly after pupae were seen, the parental generation was transferred into new rearing vials to continue egg laying. The date of appearance of newly-emerged offspring was observed for a total of 8 Cyp6z18 and 11 control rearing vials.

\section{Results}

Insecticide susceptibility status. Insecticide resistance levels following WHO susceptibility tests on F1 female mosquitoes to all six insecticides tested are shown in Fig. 2. The lowest mortality $0.97 \%$ (95\% CI $0.92-$ $5.09 \%$ ) was observed to permethrin while for DDT, lambdacyhalothrin, bendiocarb and deltamethrin the mortality rate ranged from $1.63 \%$ (95\% CI 1.35-4.76\% -3.29\% (95\% CI 2.43-6.73\%) (Fig. 2). For fenitrothion we observed the highest mortality among the insecticides tested $69.42 \%$ (95\% CI 64.27-74.16\%). To investigate the effect of exposure duration on mortality, bioassays with bendiocarb and deltamethrin were also carried out for four hours. For both insecticides, an increase in mortality was detected (Fig. 2); however, only for bendiocarb did the mortality increase significantly from $2.04 \%$ (95\% CI 1.93-10.2\%)) to 58.02\% (95\% CI 51.53-64.25\%) whilst for deltamethrin the increase was non-significant 9.09\% (95\% CI 6.12-13.59\%).

Adult mosquitoes were also assayed with bendiocarb and deltamethrin for four hours exposure after pre-exposure in parallel to three synergist compounds (TPP, DEM and PBO). Synergism was observed for both insecticides following TPP and PBO pre-exposure whilst no significant effect on mortality was detected for DEM (Fig. 2). TPP and PBO significantly increased the mortality of bendiocarb from 58.02\% (95\% CI 51.53-64.25\%) to $80.51 \%$ (95\% CI $69.6-88.34 \%)$ and $83.14 \%$ (95\% CI 73.41-89.96\%) respectively.

Frequency of Ace-1 resistant alleles in bendiocarb selected mosquitoes. Both dead and alive mosquitoes following exposure to $0.1 \%$ bendiocarb (4h) were genotyped for the Ace $1-119 \mathrm{~S}$ mutation using a custom Taqman assay. The wild-type allele was observed at the highest frequency (Fig. 3a) $86.22 \%$ (95\% CI 83.02-90.34) with homozygous genotypes predominating $72.44 \%$ (95\% CI 68.72-76.64). No homozygous resistant genotypes were detected (Fig. 3b). There was a highly significant association between the Ace1-119S allele and bendiocarb resistant phenotype (Fig. 3c; $P<0.0001$, Fisher's Exact test) with an OR of 25 (95\% CI 3.37-186).

Gene expression profiling of bendiocarb selected mosquitoes. To identify candidate genes associated with resistance in bendiocarb selected mosquitoes, transcriptomic profiles of three groups of Ace-1 wild-type (119G) mosquitoes were compared: Ugandan bendiocarb exposure survivors, Ugandan Control exposed (sympatric control) and a control exposed fully susceptible TPRI strain. Among the three groups we identified 32 probes significantly differently expressed in the ANOVA analysis applying a threshold of $-\log _{10}$ false-discovery rate (FDR) adjusted $P$ value $>2.5$, of which $50 \%$ of the probes had higher expression in the Ugandan exposed compared to both the sympatric control and TPRI (Table 1). The list of top candidate genes included two P450s: 
(a)

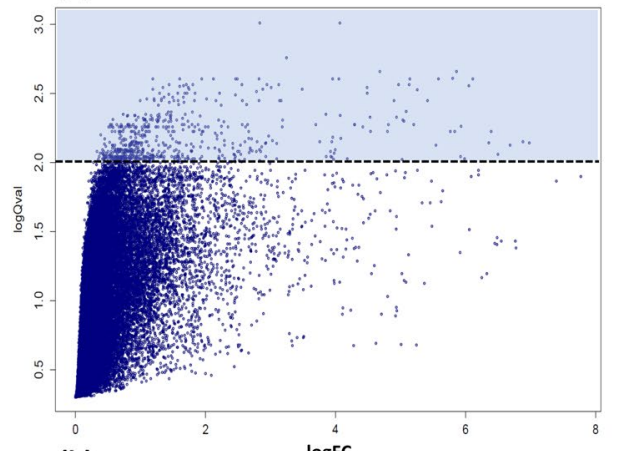

(b)

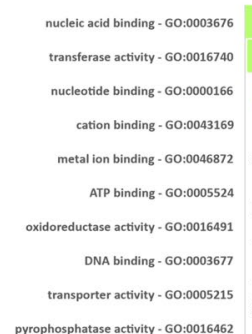

$\operatorname{logFC}$

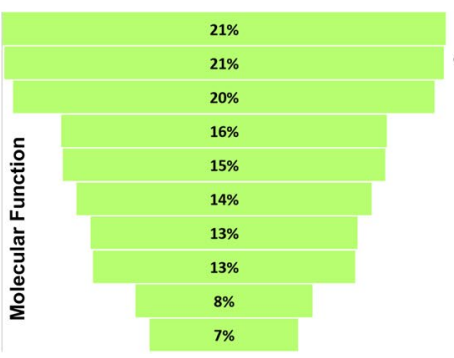

(c)

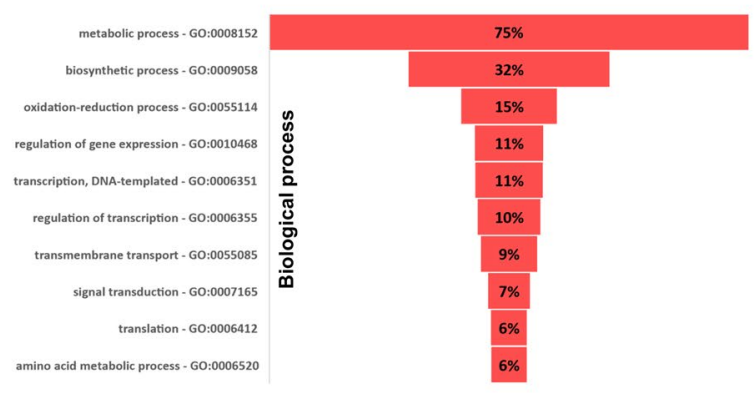

(d)

Figure 4. Candidate genes differentially transcribed in C. quinquefasciatus bendiocarb selected mosquitoes. (a) Changes of gene expression between the three groups (Uganda exposed and un-exposed and TPRI) presented as a volcano plot. (b-d) Are sunburst plots showing representative top $10 \mathrm{GO}$ term clusters (molecular function, biological process and cellular component, respectively) of differentially expressed transcripts with FDR $>2.0$.

CPIJ020018 (shown to be Cyp6z18 following reannotation - see below) and Cyp6n23, which displayed increases in gene expression compared to the TPRI strain of 8.75 and 4.37 -fold, respectively.

For functional characterization of the most significantly differentially expressed probes, a list of probes with $\mathrm{a}-\log _{10}$ false-discovery rate (FDR) $>2$ obtained from the ANOVA (Fig. $4 \mathrm{a}$ ) comparison was submitted to Gene Ontology (GO) analysis. After removing duplicate probes, 358 unique genes were submitted to VectorBase for the GO term search using Biomart (for complete annotation see Supplementary Table S3.) In total 298 terms were obtained with the majority of extracted GO terms clustered on the molecular function and biological process categories (see Supplementary Table S4 for GO Term frequencies and description). Among the top 10 enriched terms for each category, GO terms linked to metabolic process, biosynthetic process, transport, membrane, integral component of membrane, nucleus, binding, cation binding and metal ion binding were observed with percentage of annotations ranging from 2.16 to $75.39 \%$ (1-81 genes, respectively) (Fig. $4 \mathrm{~b}-\mathrm{d}$ ). Metabolic process was the predominant term across all categories corresponding to $75.39 \%$ of enriched GO terms, while within the category of molecular function and cellular component we observed a large proportion $(21.23 \%)$ of terms associated with binding functions (29 genes) and membrane 61.59\% (29 genes), respectively. Further analysis utilising the 358 unique genes showed five clusters of GO terms with significant enrichment (enrichment score >1.3: Supplementary Tables S5 and S6). Cluster 1 had the highest enrichment score (5.15) and was associated with the GO term 'transferase activity' and Interpro IDs for Glutathione S-transferases ('Glutathione S-transferase, $\mathrm{N}$-terminal' Benjamini-Hochberg $\mathrm{P}=6.7 \times 10^{-6}$; 'Glutathione S-transferase, C-terminal' Benjamini-Hochberg $\left.\mathrm{P}=2 \times 10^{-5}\right)$.

To explore further the transcriptomic profile of the bendiocarb resistant phenotype, pairwise comparison using Student's t-test was applied to compare exposed and unexposed Ugandan mosquitoes to the TPRI susceptible strain. Significantly up and down-regulated genes with a fold-change $>2.5$ were also investigated by GO analysis. For down-regulated genes we observed similar figures between exposed and unexposed mosquitoes in contrast to up-regulated genes where we identified 33 genes exclusively in the exposed mosquitoes (Fig. 5a). Pairwise comparisons also identified eight significantly differentially expressed genes putatively associated with insecticide detoxification: three exclusively in the pools of exposed mosquitoes: GSTs (CPIJ018629-RA; fold-change 2.79 and CIPJ018632-RA, fold-change 2.0) and esterase (CPIJ013918-RA; fold-change 2.86) whereas for the other five: P450 (CPIJ020018-RA), GSTs (CPIJ010814-RA, CPIJ018624-RA, CPIJ018626-RA) and esterase (CPIJ013917-RA) were observed in both Ugandan exposed and unexposed mosquitoes.

In general, the GO term enrichment of the up and down-regulated genes for the Ugandan exposed and unexposed mosquitoes shows a similar list of terms (Fig. 5b) with the exception of three terms: metabolic process, phosphate-containing compound metabolic process and cation binding that were observed exclusively on the exposed mosquitoes with frequencies of 60.61, 16.39 and $17.07 \%$, respectively (Fig. 5c). Nevertheless, this analysis excluded 39 and 18 up and down-transcribed genes (See Supplementary Tables S7 and S8), respectively, such as esterases (CPIJ013917-RA and CPIJ013918-RA) and heat shock proteins (CPIJ013880-RA and CPIJ005642) for instance, that were not associated with GO terms from VectorBase. 


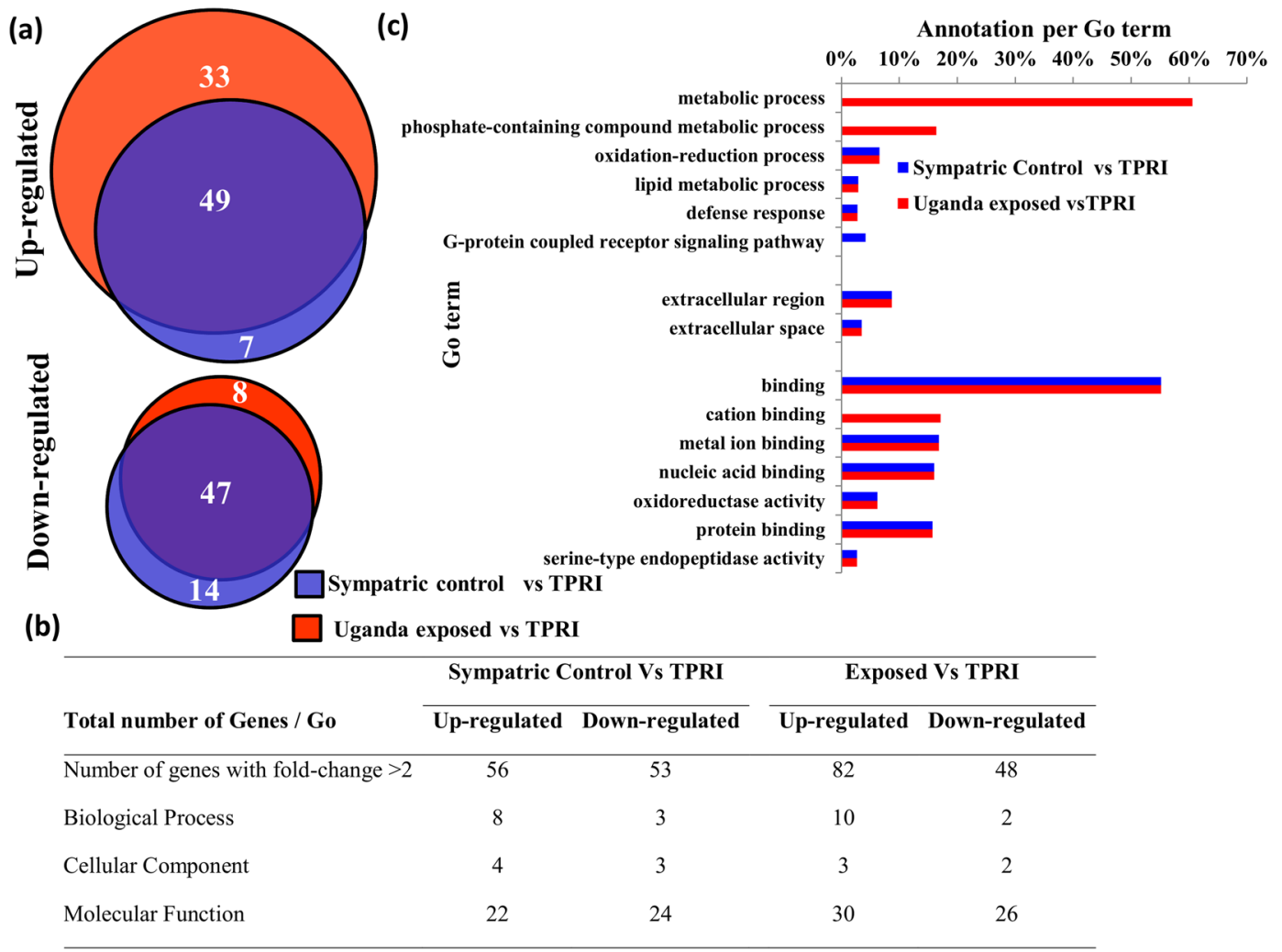

Figure 5. Transcriptomic profile of differentially expressed genes with fold-change $>2$ in Uganda exposed and sympatric mosquitoes compared to TPRI. (a) Venn diagram showing the overlap of up- and down-regulated transcripts between the three groups. (b) Comparison of the number of GO terms identified by each pair-wise comparison. (c) GO term enrichment of up-regulated transcripts between the groups with frequency higher than $2 \%$.

Candidate gene validation by qPCR. The expression patterns of three candidate genes, randomly chosen from the list of top candidates, (both P450 genes Cyp6z18 and Cyp6n23, plus R2D2) were additionally assessed by qPCR. Satisfactory PCR efficiency ranging from 91.4 to $101.8 \%$, (within the $10 \%$ acceptable variation) was obtained for primer pairs designed for candidate genes and endogenous controls (Supplementary Fig. S1). Additionally, the primer sets were specific, with a single symmetrical amplicon peak in melting curve analyses (Supplementary Fig. S2). For all three candidate genes (Cy6z17, Cyp6n23 and R2D2) we observed a good correlation between the microarray and RT-qPCR expression fold-change with ratios of up-regulation detected by both methods differing by less than $1.5 \times$ (Supplementary Fig. S3).

Annotation of CPIJ020018. After identification of CPIJ020018 as the top candidate gene in the microarray results, further analyses on the genomic and cDNA sequences available from VectorBase were conducted. This revealed an atypical gene architecture for a $\mathrm{P} 450$ gene with the presence of one intron $>3 \mathrm{~Kb}$ (Fig. 6a). Closer analysis of the genomic sequence indicated a region of $809 \mathrm{bp}$ with no nucleotide information (Ns) internal to the CPIJ020018 genomic sequence. In silico analysis of a contig constructed after PCR amplification and cloning of the complete region encompassing the genomic region of interest (accession number MH822866), suggested the presence of two distinct P450s instead of the single Cyp6z16 predicted by the automated annotation in VectorBase. The two genes predicted are each composed of two exons separated by one intron (Fig. $6 \mathrm{~b}$ and Supplementary Material 1). BLAST analysis of both predicted amino-acid sequences against Anopheles gambiae and Aedes aegypti sequences available on VectorBase and the Cytochrome P450 homepage ${ }^{54}$ shows the top hits belong to CYP genes from the $\mathrm{Z}$ family. Following submission to the $\mathrm{P} 450$ nomenclature database the partial $\mathrm{P} 450$ is now labelled cyp6z16 and the full-length novel P450, which is interrogated by the microarray probes (hitting CPIJ020018 exon-2) is formally labelled cyp6z18 (note cyp6z17 is annotated is annotated on supercontig 3.3058). Together the gene prediction and BLAST analysis carried out indicate that the top candidate gene from the microarray analysis is cyp6z18 and not cyp $6 z 16$ (which is not interrogated by any probes due to incorrect annotation). cyp6z18 is closely related to other P450s which have previously been associated with metabolic resistance in both An. gambiae ${ }^{55,56}$ and Ae. aegypti ${ }^{57,58}$ (Fig. 6c). We note that whilst our microarray design was undertaken on the CpipJ1 assembly, the CpipJ2 assembly (available from April 2014) did not revise the assembly or annotation of this scaffold.

Transgenic expression of cyp6z18 in Drosophila flies. Drosophila melanogaster flies were transformed with a transgene encoding cyp6z18 for expression using the GAL4/UAS system. Individuals containing the 

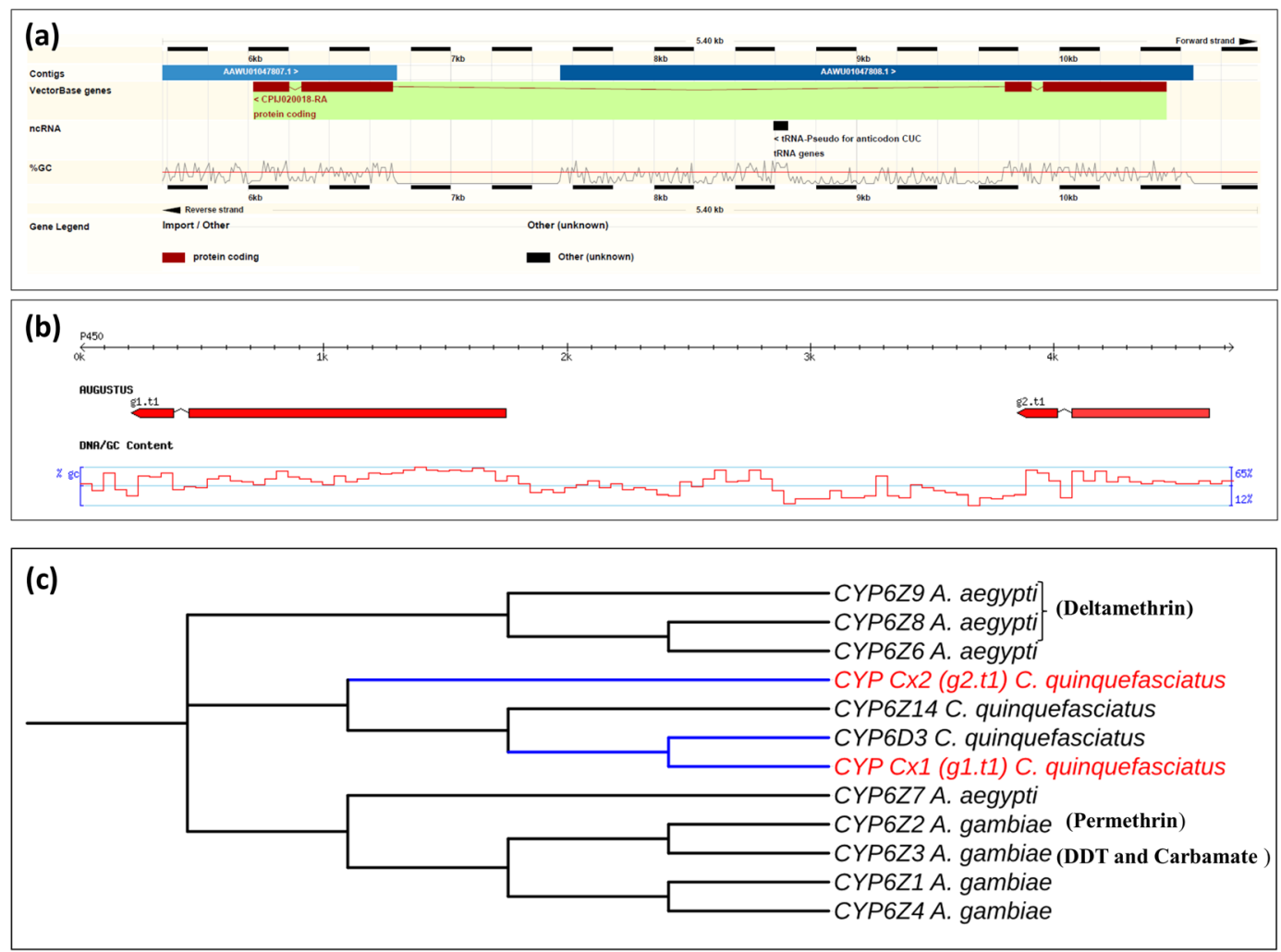

Figure 6. Cyp6z16 and cyp6z18 predicted gene structure and annotation. (a) output of the VectorBase genome Browser suggesting a gene architecture with four exons and three introns. Figure adapted from VectorBase ${ }^{96}(\mathbf{b})$ Schematic representation of CPIJ020018 after re-annotation using Augustus software, indicating two distinct genes here named cyp6z18 (g1.t1) and cyp6z16 (g2.t1). (c) Unrooted distance neighbour joining tree showing phylogenetic relationship of the predicted gene cyp6z18 from C. quinquefasciatus to Aedes aegypti and An. gambiae cytochrome P450s from the CYP6 gene family. Blue branches and genes highlighted in red represents the relationship of the re-annotated gene structure.

cyp6z18 gene and untransformed controls were crossed to flies of the Act5C-GAL4 driver line to induce ubiquitous expression of cyp6z18. Expression of cyp6z18 negatively impacted fly fitness, evident in prolonged pre-adult development. The number of days between placing adult flies in a rearing vial and the first offspring emerging was measured. A significant difference in this value was detected, with an average of 23 days for cyp6z18-expressing flies (95\% CI 21.21-25.04) and 16 days for untransformed control flies (95\% CI 15.23-16.41) (unpaired t-test with Welch's correction $P<0.0001, N=19$ ). Because of this fitness cost, experiments to test the impact of cyp6z18 expression upon insecticide resistance were not pursued.

\section{Discussion}

Insecticide resistance is widespread in malaria vectors in Uganda ${ }^{37,59-63}$ and known to result from both target-site and detoxification mechanisms ${ }^{63-65}$. In this study, we investigated the insecticide susceptibility status and possible mechanisms involved with insecticide resistance in the LF vector C. quinquefasciatus mosquitoes collected in a region of Uganda (Tororo) where both Anopheles gambiae and Anopheles funestus exhibit resistance to the insecticides currently used for malaria control ${ }^{37,62,63}$. We have shown previously ${ }^{41}$ that the pattern of target-site mutations in Ugandan Culex varies regionally despite intense gene flow, suggesting a heterogeneous pattern of insecticidal selection pressures. Here we show that in Culex from Tororo, eastern Uganda, resistance to three of the four classes of insecticide occurs at high levels and, at least for bendiocarb, is mediated by both target-site and metabolic mechanisms. Although, the G119S mutation in Ace-1 is not at high frequency, it is strongly associated with resistance with an OR of 25 (95\% CI 3.37-186). With such a strong resistance association it seems surprising that the $119 \mathrm{~S}$ allele is at such low frequency $14 \%$ (95\% CI 7.9-22.4) in the Nagongera population. This is a much lower frequency than observed in other vector mosquitoes where carbamates are routinely used in vector control ${ }^{66}$. Whilst insecticides linked to selection of Ace-1 resistant alleles have not been officially applied for vector control in the studied area for over one decade ${ }^{67,68}$ indoor residual spraying (IRS) using bendiocarb was conducted in Tororo from December 2014 - February 2015 $5^{69,70}$, after the period of collection of these samples, and hence this allele frequency is higher than expected. Nevertheless, an indirect source of insecticide exposure due to agricultural activities could also be shaping the insecticide resistance as described for C. quinquefasciatus populations from $\operatorname{Iran}^{71}$. However, the Ace-1 mutation is known to confer a fitness disadvantage which may explain the low frequency of the resistance associated allele ${ }^{72}$. This recent evolution of insecticide resistance in Ugandan Culex populations, possibly in response to malaria vector control, has also been suggested in other 
African countries such as Tanzania and Zambia, where moderate resistance has also been detected to bendiocarb, in contrast to the high levels of resistance detected to both pyrethroids and DDT ${ }^{16,73}$.

The potential involvement of metabolic resistance in bendiocarb resistant C. quinquefasciatus from Tororo is supported by the synergist assays, which show an increase in mortality to bendiocarb when mosquitoes were pre-exposed to either TPP (a synergist of esterases) or PBO (a synergist of cytochrome P450s). Together the Ace-1 genotyping and synergist assay data strongly suggested an alternative mechanism of resistance to the well-known Ace1-119S target-site mutation in the Ugandan bendiocarb resistant phenotype. Transcriptomic profiling of Ace1 wild-type mosquitoes identified two P450s (CPIJ020018 and Cyp6n23) with the highest up-regulation in the resistant samples among the top candidate genes. Over-expression of both these cytochrome P450s (CPIJ020018 and cyp6n23) identified in our analysis are especially relevant as many genes belonging to this gene family have been associated with insecticide metabolism in a variety of vector species $28,74,75$.

The likely association of this candidate cytochrome $\mathrm{P} 450 \mathrm{~s}$ with the carbamate resistant phenotype is supported also by the synergism effect of the $\mathrm{P} 450$ inhibitor $\mathrm{PBO}^{76}$. Most CYPs previously associated with the insecticide resistance phenotype in mosquitoes, (e.g. cyp6p3, cyp9j32 and cyp6m10) are typically associated with metabolism of pyrethroids and DDT ${ }^{56,75,77}$, while so far very few examples of bendiocarb metabolism by P450 have been reported. Recently, Edi et al. ${ }^{78}$ demonstrated that cyp $6 p 3$ is associated with the bendiocarb resistant phenotype in An. gambiae from Tiassalé, Cote d'Ivoire and confirmed its capability to metabolise bendiocarb. Additionally, two other An. gambiae P450s (cyp6z1 and cyp6z2) have also been demonstrated to be capable of metabolizing the carbamate insecticide carbaryl ${ }^{79}$ and upregulation of the An. funestus cyp $6 z 1$ is associated with carbamate resistance in this species ${ }^{80}$. Interestingly, both cytochrome P450s identified here belong to the CYP6 family, which includes most of the CYPs genes already described as insecticide metabolizers ${ }^{23}$. Our GO term enrichment analysis also indicated a likely impact of other metabolic genes such as GSTs, which from our data had the highest enrichment score in the cluster analysis, with two GST genes (CPIJ018629-RA and CIPJ018632-RA) overexpressed exclusively in exposed mosquitoes. These findings indicate that further functional analysis should be performed to pinpoint the role of GSTs in the carbamate resistant-phenotype of Culex. We note that other studies have already shown that genes belonging to this family have roles in resistance to DDT and pyrethroids in Culex mosquitoes $^{81,82}$.

For the candidate gene cyp6z18, functional enzymatic characterization for insecticide metabolism, was applied as an in vivo approach using transgenic gene expression in Drosophila. Unfortunately, this approach did not succeed in validating this gene as the transgenic construction negatively affected Drosophila development. Further work using alternative approaches such as in vivo gene expression through CRISPR/Cas $9^{83}$ or in vitro metabolism assays e.g. ${ }^{28}$ could be applied to confirm the role of the candidate gene in metabolic resistance. As shown herein and elsewhere ${ }^{84,85}$, investigation of resistant phenotypes in light of metabolic resistance is imperative for pinpointing the genetic mechanisms associated with evolving insecticide resistance. For instance, while our data show that both target-site and detoxification gene over-expression underly the resistance to bendiocarb in $C$. quinquefasciatus from Tororo, other studies have demonstrated that $\mathrm{P} 450$ overexpression alone is the primary mechanism in $k d r$-free populations such as in Anopheles arabiensis from Chad, Central Africa ${ }^{84}$ and Malaysian Aedes albopictus ${ }^{85}$. Taken together, these findings highlight the relevance of identifying the mechanisms driving resistance in order to facilitate the development of field-applicable markers to detect increased resistance at the early stages of resistance development as well as to assist in decision-making for more effective control interventions. For instance, choosing either insecticide-only nets or insecticidal-synergistic nets (e.g. those incorporating piperonyl butoxide - $\mathrm{PBO})^{86}$ or the rational use of insecticides from distinct classes to tackle the threat of an increased burden of vector-borne diseases driven by reduced efficacy of ongoing vector control interventions ${ }^{87}$.

Further analysis of the genomic and transcriptomic sequences of the top candidate gene CPIJ020018 (annotated as cyp6z16 in VectorBase) suggests annotation inaccuracy. After re-annotation, our analysis indicates that CPIJ020018 consists of two distinct CYP genes (cyp6z16 and cyp6z18) instead of one as suggested by the automated annotation of VectorBase. Annotation problems as observed for cyp $6 z 16$ may potentially occur for other genes ( $\mathrm{see}^{88}$ for annotation deficiencies of recent Anopheles sequencing), thus a review and improvement of gene annotation for C. quinquefasciatus and other vector species such as Anopheles dirus, Aedes albopictus and Lutzomyia longipalpis recently included on the VectorBase genome browser, especially of gene families linked to insecticide resistance, would benefit future transcriptomic monitoring of insecticide resistance. Whilst, the increased usage of RNA-Seq for differential gene expression analyses may suggest this is unnecessary, the typical RNA-Seq pipeline maps reads to annotated gene sets and hence a robust annotation of these gene families would still be beneficial. C. quinquefasciatus remains the poor relation of disease vectors with respect to assembly and annotation of genome sequence, whilst the genome sequences of A. gambiae and A. aegypti have received important and successful genome updates ${ }^{89,90}$ Culex remains assembled with a high number of scaffolds and hence gaps.

This microarray study also identified that four other detoxification genes (three glutathione S-transferases (GSTs) and one esterase) were also over-transcribed compared to TPRI, although they were not differently expressed between Tororo resistant and sympatric unexposed controls. High expression of these genes alone or in combination with other detoxification enzymes could also be a possible mechanism associated with the bendiocarb phenotype as reported previously ${ }^{91,92}$, although in our data no significant synergist effect by DEM, a GST inhibitor was observed, further characterization of these genes is warranted.

Although C. quinquefasciatus is a vector of important neglected tropical diseases, planning and management of vector control strategies for Anopheles species has received considerably more attention and funding. Whilst this could reflect that MDA is currently the primary intervention for LF eradication with vector control deemed secondary, a reduction of LF transmission by Culex species due to control programs aimed at anopheline species has been demonstrated ${ }^{93,94}$. Thus, in a possible integrated vector-control scenario the monitoring of insecticide resistance and determination of the mechanisms resistance for vector species of apparent secondary interest can be critical to effective integration ${ }^{95}$. 


\section{Conclusions}

Our data demonstrate that although Ugandan C. quinquefasciatus mosquitoes had not been covered by a specific, targeted local vector control program, high levels of insecticide resistance were identified in the studied population, indicating that application of insecticide to control other species with public health importance such as anophelines through indoor residual spray (IRS) and insecticide treated nets (ITNs), or through application of insecticides for agricultural purposes could be driving the evolution of insecticide resistance in this population. This study also provides strong evidence that a metabolic mechanism is associated with the bendiocarb resistant phenotype observed in Tororo. Lastly, by a whole-transcriptomic analysis we identify two new candidate genes belonging to the cytochrome CPY6 gene family associated with metabolic resistance to bendiocarb.

\section{References}

1. Bockarie, M. J., Pedersen, E. M., White, G. B. \& Michael, E. Role of vector control in the global program to eliminate lymphatic filariasis. Annu. Rev. Entomol. 54, 469-487 (2009).

2. de Souza, D. K. et al. Diversity and transmission competence in lymphatic filariasis vectors in West Africa, and the implications for accelerated elimination of Anopheles-transmitted filariasis. Parasit. Vectors 5, 259 (2012).

3. Katiyar, D. \& Singh, K. L. Filariasis: current status, treatment and recent advances in drug development. Curr. Med. Chem. 18, 2174-2185 (2011).

4. Rebollo, M. P. \& Bockarie, M. J. Toward the elimination of lymphatic filariasis by 2020 : treatment update and impact assessment for the endgame. Expert Rev. Anti. Infect. Ther. 11, 723-731 (2013).

5. van den Berg, H., Kelly-Hope, L. A. \& Lindsay, S. W. Malaria and lymphatic filariasis: the case for integrated vector management. The Lancet Infect. Diseases 13, 89-94 (2013).

6. Ichimori, K. et al. Global Programme to Eliminate Lymphatic Filariasis: the processes underlying programme success. PLoS Negl. Trop. Dis. 8, E3328 (2014).

7. Gyapong, J. O. \& Twum-Danso, N. A. Y. Global elimination of lymphatic filariasis: fact or fantasy? Trop. Med. Int. Health 11, 125-128 (2006).

8. Sunish, I. P. et al. Vector control complements mass drug administration against Bancroftian filariasis in Tirukoilur, India. Bull. World Health Organ. 85, 138-145 (2007).

9. Nkya, T. E., Akhouayri, I., Kisinza, W. \& David, J.-P. Impact of environment on mosquito response to pyrethroid insecticides: facts, evidences and prospects. Insect Biochem. Mol. Biol. 43, 407-416 (2013).

10. Rivero, A., Vezilier, J., Weill, M., Read, A. F. \& Gandon, S. Insecticide control of vector-borne diseases: when is insecticide resistance a problem? PLoS Pathog. 6, e1001000 (2010).

11. Nauen, R. Insecticide resistance in disease vectors of public health importance. Pest Manag. Sci. 63, 628-633 (2007).

12. Sparks, T. C. Insecticide discovery: an evaluation and analysis. Pestic. Biochem. Physiol. 107, 8-17 (2013).

13. Feyereisen, R., Dermauw, W. \& Van Leeuwen, T. Genotype to phenotype, the molecular and physiological dimensions of resistance in arthropods. Pestic. Biochem. Physiol. 121, 61-77 (2015).

14. Kioulos, I., Kampouraki, A., Morou, E., Skavdis, G. \& Vontas, J. Insecticide resistance status in the major West Nile virus vector Culex pipiens from Greece. Pest Manag. Sci. 70, 623-627 (2014).

15. Martins, W. F. S. et al. Detection and quantitation of copy number variation in the voltage-gated sodium channel gene of the mosquito Culex quinquefasciatus. Sci. Rep. 7, 5821 (2017).

16. Jones, C. M. et al. Insecticide resistance in Culex quinquefasciatus from Zanzibar: implications for vector control programmes. Parasit. Vectors 5 (2012).

17. Zhao, M. et al. Point mutations associated with organophosphate and carbamate resistance in Chinese strains of Culex pipiens quinquefasciatus (Diptera: Culicidae). PLoS ONE 9, e95260 (2014).

18. Martins, W. F. S. et al. Development and application of a tri-allelic PCR assay for screening Vgsc-L1014F $k d r$ mutations associated with pyrethroid and organochlorine resistance in the mosquito Culex quinquefasciatus. Parasit. Vectors 12, 232 (2019).

19. David, J. P., Ismail, H. M., Chandor-Proust, A. \& Paine, M. J. I. Role of cytochrome P450s in insecticide resistance: impact on the control of mosquito-borne diseases and use of insecticides on Earth. Philos. Trans. R. Soc. Lond. B Biol. Sci. 368 (2013).

20. Li, X., Schuler, M. A. \& Berenbaum, M. R. Molecular mechanisms of metabolic resistance to synthetic and natural xenobiotics. Annu. Rev. Entomol. 52, 231-253 (2007).

21. Pavlidi, N., Vontas, J. \& Van Leeuwen, T. The role of glutathione S-transferases (GSTs) in insecticide resistance in crop pests and disease vectors. Current Opin. Insect Sci. 27, 97-102 (2018).

22. Weetman, D., Djogbenou, L. S. \& Lucas, E. Copy number variation (CNV) and insecticide resistance in mosquitoes: evolving knowledge or an evolving problem? Current Opin. Insect Sci. 27, 82-88 (2018).

23. Hemingway, J., Hawkes, N. J., McCarroll, L. \& Ranson, H. The molecular basis of insecticide resistance in mosquitoes. Insect Biochem. Mol. Biol. 34, 653-665 (2004).

24. Wilding, C. S. Regulating resistance: CncC:Maf, antioxidant response elements and the overexpression of detoxification genes in insecticide resistance. Current Opin. Insect Sci. 27, 89-96 (2018).

25. Wilding, C. S. et al. A cis-regulatory sequence driving metabolic insecticide resistance in mosquitoes: functional characterisation and signatures of selection. Insect Biochem. Mol. Biol. 42, 699-707 (2012).

26. Sun, X.H. et al. A novel miRNA, miR-13664, targets CPCYP314A1 to regulate deltamethrin resistance in Culex pipiens pallens. Parasitol., 1-9 (2018).

27. Bonizzoni, M. et al. RNA-seq analyses of blood-induced changes in gene expression in the mosquito vector species, Aedes aegypti. BMC Genomics 12, 82 (2011).

28. Mitchell, S. N. et al. Identification and validation of a gene causing cross-resistance between insecticide classes in Anopheles gambiae from Ghana. Proc. Natl Acad. Sciences USA 109, 6147-6152 (2012).

29. Riveron, J. M. et al. The highly polymorphic CYP6M7 cytochrome P450 gene partners with the directionally selected CYP6P9a and CYP6P9b genes to expand the pyrethroid resistance front in the malaria vector Anopheles funestus in Africa. BMC Genomics 15 (2014).

30. Farajollahi, A., Fonseca, D. M., Kramer, L. D. \& Kilpatrick, A. M. "Bird biting" mosquitoes and human disease: a review of the role of Culex pipiens complex mosquitoes in epidemiology. Infect. Genet. Evol. 11, 1577-1585 (2011).

31. Komagata, O., Kasai, S. \& Tomita, T. Overexpression of cytochrome P450 genes in pyrethroid-resistant Culex quinquefasciatus. Insect Biochem. Mol. Biol. 40, 146-152 (2010).

32. Liu, N., Li, T., Reid, W.R., Yang, T. \& Zhang, L. Multiple cytochrome P450 genes: Their constitutive overexpression and permethrin induction in insecticide resistant mosquitoes, Culex quinquefasciatus. PLoS ONE 6 (2011).

33. Reid, W. R., Zhang, L., Gong, Y., Li, T. \& Liu, N. Gene expression profiles of the Southern house mosquito Culex quinquefasciatus during exposure to permethrin. Insect Sci. 25, 439-453 (2018).

34. Li, C.-X. et al. Identification of genes involved in pyrethroid-, propoxur-, and dichlorvos- insecticides resistance in the mosquitoes, Culex pipiens complex (Diptera: Culicidae). Acta Trop. 157, 84-95 (2016). 
35. Kudom, A. A., Mensah, B. A. \& Nunoo, J. Assessment of anti mosquito measures in households and resistance status of Culex species in urban areas in southern Ghana: Implications for the sustainability of ITN use. Asian Pacific J. Trop. Med. 6, 859-864 (2013).

36. Raouf, S. et al. Resurgence of malaria following discontinuation of indoor residual spraying of insecticide in an area of Uganda with previously high-transmission intensity. Clin. Infect. Dis. 65, 453-460 (2017).

37. Morgan, J. C., Irving, H., Okedi, L. M., Steven, A. \& Wondji, C. S. Pyrethroid resistance in an Anopheles funestus population from Uganda. PLoS ONE 5 (2010).

38. Smith, J. L. \& Fonseca, D. M. Rapid assays for identification of members of the Culex (Culex) pipiens complex, their hybrids, and other sibling species (Diptera: Culicidae). Am. J. Trop. Med. Hyg. 70, 339-345 (2004).

39. Oxborough, R. M. et al. ITN mixtures of chlorfenapyr (pyrrole) and alphacypermethrin (pyrethroid) for control of pyrethroid resistant Anopheles arabiensis and Culex quinquefasciatus. PLoS ONE 8, e55781 (2013).

40. WHO. Test procedures for insecticide resistance monitoring in malaria vector mosquitoes. (2013).

41. Martins, W. F. S. et al. Local selection in the presence of high levels of gene flow: evidence of heterogeneous insecticide selection pressure across Ugandan Culex quinquefasciatus populations. PLoS Negl. Trop. Dis. 11, e0005917 (2017).

42. Arensburger, P. et al. Sequencing of Culex quinquefasciatus establishes a platform for mosquito comparative genomics. Science 330, $86-88(2010)$

43. Kasai, S., Komagata, O., Okamura, Y. \& Tomita, T. Alternative splicing and developmental regulation of glutathione transferases in Culex quinquefasciatus Say. Pestic. Biochem. Physiol. 94, 21-29 (2009).

44. Vinciotti, V. et al. An experimental evaluation of a loop versus a reference design for two-channel microarrays. Bioinformatics 21, 492-501 (2005).

45. R Core Team. R: a language and environment for statistical computing (R Foundation for Statistical Computing, Vienna, Austria, 2012).

46. Smyth, G. K. Limma: linear models for microarray data. In Bioinformatics and Computational Biology Solutions Using R and Bioconductor (eds R. Gentleman et al.) 397-420 (Springer-Verlag, 2005).

47. Wu, H., Kerr, M. K., Cui, X. \& Churchill, G. A. MAANOVA: a software package for the analysis of spotted cDNA microarray experiments. In The Analysis of Gene Expression Data: Methods and Software (eds Parmigiani, G., Garett, E. S., Irizarry, R. A. \& Zeger, S. L.) 313-341 (Springer-Verlag, 2003).

48. Supek, F., Bošnjak, M., Škunca, N. \& Šmuc, T. REVIGO summarizes and visualizes long lists of gene ontology terms. PLoS ONE 6, e21800 (2011).

49. Huang, D. W., Sherman, B. T. \& Lempicki, R. A. Systematic and integrative analysis of large gene lists using DAVID bioinformatics resources. Nat. Protoc. 4, 44 (2009).

50. Huang, D. W., Sherman, B. T. \& Lempicki, R. A. Bioinformatics enrichment tools: paths toward the comprehensive functional analysis of large gene lists. Nucleic Acids Res. 37, 1-13 (2009).

51. Rozen, S. \& Skaletsky, H. Primer3 on the WWW for general users and for biologist programmers. Methods Mol. Biol. 132, 365-386 (2000).

52. Livak, K. J. \& Schmittgen, T. D. Analysis of relative gene expression data using real-time quantitative PCR and the $2^{\text {-Delta Delta CT }}$ method. Methods 25, 402-408 (2001).

53. Stanke, M. \& Morgenstern, B. AUGUSTUS: a web server for gene prediction in eukaryotes that allows user-defined constraints. Nucleic Acids Res. 33, W465-W467 (2005).

54. Nelson, D. R. The Cytochrome P450 Homepage. Hum. Genomics 4, 59-65 (2009).

55. David, J.-P. et al. The Anopheles gambiae detoxification chip: a highly specific microarray to study metabolic-based insecticide resistance in malaria vectors. Proc. Natl. Acad. Sci. USA 102, 4080-4084 (2005).

56. Müller, P. et al. Field-caught permethrin-resistant Anopheles gambiae overexpress CYP6P3, a P450 that metabolises pyrethroids. PLoS Genet. 4, e1000286 (2008).

57. Marcombe, S. et al. Insecticide resistance in the dengue vector Aedes aegypti from Martinique: distribution, mechanisms and relations with environmental factors. PLoS ONE 7, e30989 (2012).

58. Marcombe, S. et al. Exploring the molecular basis of insecticide resistance in the dengue vector Aedes aegypti: a case study in Martinique Island (French West Indies). BMC Genomics 10, 494 (2009).

59. Abeku, T. A. et al. Insecticide resistance patterns in Uganda and the effect of indoor residual spraying with bendiocarb on $k d r$ L1014S frequencies in Anopheles gambiae s.s. Malar. J. 16, 156 (2017).

60. Okia, M. et al. Insecticide resistance status of the malaria mosquitoes: Anopheles gambiae and Anopheles funestus in eastern and northern Uganda. Malar. J. 17, 157 (2018).

61. Yeka, A. et al. Malaria in Uganda: challenges to control on the long road to elimination: I. Epidemiology and current control efforts. Acta Trop. 121, 184-195 (2012).

62. Mawejje, H. D. et al. Insecticide resistance monitoring of field-collected Anopheles gambiae s.l. populations from Jinja, eastern Uganda, identifies high levels of pyrethroid resistance. Med. Vet. Entomol. 27, 276-283 (2013).

63. Wilding, C. et al. Parallel evolution or purifying selection, not introgression, explains similarity in the pyrethroid detoxification linked GSTE4 of Anopheles gambiae and An. arabiensis. Mol. Genet. Genomics 290, 201-215 (2014).

64. Weetman, D. et al. Candidate-gene based GWAS identifies reproducible DNA markers for metabolic pyrethroid resistance from standing genetic variation in East African Anopheles gambiae. Sci. Rep. 8, 2920 (2018).

65. Ramphul, U. et al. Insecticide resistance and its association with target-site mutations in natural populations of Anopheles gambiae from eastern Uganda. Trans. R. Soc. Trop. Med. Hyg. 103, 1121-1126 (2009).

66. Edi, C. V. A., Koudou, B. G., Jones, C. M., Weetman, D. \& Ranson, H. Multiple-insecticide resistance in Anopheles gambiae mosquitoes, Southern Cote d'Ivoire. Emerg. Infect. Dis. 18, 1508-1511 (2012).

67. Kigozi, R. et al. Indoor residual spraying of insecticide and malaria morbidity in a high transmission intensity area of Uganda. PLoS ONE 7, e42857 (2012).

68. Yeka, A. et al. Malaria in Uganda: challenges to control on the long road to elimination I. Epidemiology and current control efforts. Acta Trop. 121, 184-195 (2012).

69. Katureebe, A. et al. Measures of malaria burden after long-lasting insecticidal net distribution and indoor residual spraying at three sites in Uganda: a prospective observational study. PLoS Med. 13, e1002167 (2016).

70. Muhindo, M. K. et al. Reductions in malaria in pregnancy and adverse birth outcomes following indoor residual spraying of insecticide in Uganda. Malar. J. 15, 437 (2016).

71. Vatandoost, H. et al. Enhanced tolerance of house mosquito to different insecticides due to agricultural and household pesticides in sewage system of Tehran, Iran. Iranian J. Env. Health Sci. Eng. 1, 46-50 (2004).

72. Weetman, D. et al. Contemporary evolution of resistance at the major insecticide target site gene Ace- 1 by mutation and copy number variation in the malaria mosquito Anopheles gambiae. Mol. Ecol. 24, 2656-2672 (2015).

73. Norris, L. C. \& Norris, D. E. Insecticide resistance in Culex quinquefasciatus mosquitoes after the introduction of insecticide-treated bed nets in Macha, Zambia. J. Vector Ecol. 36, 411-420 (2011).

74. Hardstone, M. C., Komagata, O., Kasai, S., Tomita, T. \& Scott, J. G. Use of isogenic strains indicates CYP9M10 is linked to permethrin resistance in Culex pipiens quinquefasciatus. Insect Mol. Biol. 19, 717-726 (2010).

75. Strode, C. et al. Genomic analysis of detoxification genes in the mosquito Aedes aegypti. Insect Biochem. Mol. Biol. 38, 113-123 (2008). 
76. Feyereisen, R. Insect CYP genes and P450 enzymes. In Insect Molecular Biology and Biochemistry (ed. Gilbert, L. I.) 236-316 (2012).

77. Djouaka, R. F. et al. Expression of the cytochrome P450s, CYP6P3 and CYP6M2 are significantly elevated in multiple pyrethroid resistant populations of Anopheles gambiae s.s. from Southern Benin and Nigeria. BMC Genomics 9 (2008).

78. Edi, C. V. et al. CYP6 P450 enzymes and ACE-1 duplication produce extreme and multiple insecticide resistance in the malaria mosquito Anopheles gambiae. PLoS Genet. 10 (2014).

79. Chiu, T.-L., Wen, Z., Rupasinghe, S. G. \& Schuler, M. A. Comparative molecular modeling of Anopheles gambiae CYP6Z1, a mosquito P450 capable of metabolizing DDT. Proc. Natl. Acad. Sci. USA 105, 8855-8860 (2008).

80. Ibrahim, S. S., Ndula, M., Riveron, J. M., Irving, H. \& Wondji, C. S. The P450 CYP6Z1 confers carbamate/pyrethroid cross-resistance in a major African malaria vector beside a novel carbamate-insensitive N485I acetylcholinesterase-1 mutation. Mol. Ecol. 25, 3436-3452 (2016).

81. Samra, A. I., Kamita, S. G., Yao, H.-W., Cornel, A. J. \& Hammock, B. D. Cloning and characterization of two glutathione S-transferases from pyrethroid-resistant Culex pipiens. Pest Manag. Sci. 68, 764-772 (2012).

82. Wang, W. et al. Over-transcription of genes in a parathion-resistant strain of mosquito Culex pipiens quinquefasciatus. Insect Sci. 22, $150-156(2015)$

83. Taning, C. N. T., Van Eynde, B., Yu, N., Ma, S. \& Smagghe, G. CRISPR/Cas9 in insects: applications, best practices and biosafety concerns. J. Insect Physiol. 98, 245-257 (2017).

84. Ibrahim, S. S., Riveron, J. M., Stott, R., Irving, H. \& Wondji, C. S. The cytochrome P450 CYP6P4 is responsible for the high pyrethroid resistance in knockdown resistance-free Anopheles arabiensis. Insect Biochem. Mol. Biol. 68, 23-32 (2016).

85. Ishak, I. H. et al. The Cytochrome P450 gene CYP6P12 confers pyrethroid resistance in $k d r$-free Malaysian populations of the dengue vector Aedes albopictus. Sci. Rep. 6, 24707 (2016).

86. Awolola, S. T. et al. Impact of PermaNet 3.0 on entomological indices in an area of pyrethroid resistant Anopheles gambiae in southwestern Nigeria. Parasit. Vectors 7, 236 (2014).

87. Fernandes, J. N., Moise, I. K., Maranto, G. L. \& Beier, J. C. Revamping mosquito-borne disease control to tackle future threats. Trends Parasitol. 34, 359-368 (2018).

88. Neafsey, D. E. et al. Highly evolvable malaria vectors: The genomes of 16 Anopheles mosquitoes. Science 347, 1258522 (2015).

89. Sharakhova, M. V. et al. Update of the Anopheles gambiae PEST genome assembly. Genome Biol. 8, R5 (2007).

90. Matthews, B. J. et al. Improved reference genome of Aedes aegypti informs arbovirus vector control. Nature 563, 501-507 (2018).

91. Reddy, B. N., Rao, B. P., Prasad, G. \& Raghavendra, K. Identification and classification of detoxification enzymes from Culex quinquefasciatus (Diptera: Culicidae). Bioinformation 8, 430-436 (2012).

92. Yan, L. et al. Transcriptomic and phylogenetic analysis of Culex pipiens quinquefasciatus for three detoxification gene families. BMC Genomics 13 (2012).

93. Pedersen, E. M. \& Mukoko, D. A. Impact of insecticide-treated materials on filaria transmission by the various species of vector mosquito in Africa. Ann. Trop. Med. Parasitol. 96, S91-S95 (2002).

94. WHO. Lymphatic filariasis: a handbook of practical entomology for national lymphatic filariasis elimination programmes (2013).

95. Pocquet, N. et al. Insecticide resistance in disease vectors from Mayotte: an opportunity for integrated vector management. Parasit. Vect. 7 (2014).

96. Giraldo-Calderón, G. I. et al. VectorBase: an updated bioinformatics resource for invertebrate vectors and other organisms related with human diseases. Nucleic Acids Res. 43, D707-D713 (2014).

\section{Acknowledgements}

We would like to thank Henry Mawejje and Chris Clarkson for supporting the field-collections in Uganda. Sample collection was supported by Award Number U19AI089674 from the National Institute of Allergy and Infectious Diseases (NIAID). Genomic and transcriptomic studies were supported by Award Number BEX-6190/10-3 from Coordination for the Improvement of Higher Education Personnel (CAPES). WFSM is a Wellcome Trust Fellow - Training Fellowship in Public Health and Tropical Medicine (Reference Number - 209305/Z/17/Z). KM was funded by the National Institutes of Health/National Institute for Allergy and Infectious Diseases (Grant Numbers HHSN266200400039C; HHSN272200900039C). We thank Dr Johannes Bischof, Institute of Molecular Life Sciences, University of Zurich for the plasmid.

\section{Author Contributions}

W.F.S.M., C.S.W. and M.J.D. conceived and designed the experiments. W.F.S.M., E.J.R., C.S.W. and A.T.I. performed the experiments. C.S.W. and K.M. designed the array. W.F.S.M., C.S.W. and M.J.D. analysed the data. C.S.W. and M.J.D. Contributed reagents/materials/sample collections. W.F.S.M. wrote the paper. C.S.W. and M.J.D., reviewed the final manuscript.

\section{Additional Information}

Supplementary information accompanies this paper at https://doi.org/10.1038/s41598-019-47850-w.

Competing Interests: The authors declare no competing interests.

Publisher's note: Springer Nature remains neutral with regard to jurisdictional claims in published maps and institutional affiliations.

(c) (i) Open Access This article is licensed under a Creative Commons Attribution 4.0 International License, which permits use, sharing, adaptation, distribution and reproduction in any medium or format, as long as you give appropriate credit to the original author(s) and the source, provide a link to the Creative Commons license, and indicate if changes were made. The images or other third party material in this article are included in the article's Creative Commons license, unless indicated otherwise in a credit line to the material. If material is not included in the article's Creative Commons license and your intended use is not permitted by statutory regulation or exceeds the permitted use, you will need to obtain permission directly from the copyright holder. To view a copy of this license, visit http://creativecommons.org/licenses/by/4.0/.

(c) The Author(s) 2019 